\title{
Effect of lung volume on the oesophageal diaphragm EMG assessed by magnetic phrenic nerve stimulation
}

\author{
Y.M. Luo, R.A. Lyall, M.L. Harris, P. Hawkins, N. Hart, M.I. Polkey, J. Moxham
}

\begin{abstract}
Effect of lung volume on the oesophageal diaphragm EMG assessed by magnetic phrenic nerve stimulation. Y.M. Luo, R.A. Lyall, M.L. Harris, P. Hawkins, N. Hart, M.I. Polkey, J. Moxham. (C) ERS Journals Ltd 2000.

ABSTRACT: Previous studies have shown conflicting results on the effect of lung volume on the diaphragm compound muscle action potential (CMAP). Consequently, the ability to quantify the oesophageal diaphragm electromyography (EMG) has been questioned. If lung volume changes have little effect on the diaphragm CMAP the accurate measurement of voluntary EMG, as an index of respiratory drive, may be possible. Furthermore, the measurement of CMAP could provide useful clinical information when evaluating patients with neuromuscular disease.

To reassess the effect of lung volume on the oesophageal diaphragm CMAP, six normal subjects were studied using an oesophageal catheter incorporating seven electrodes (number one being proximal and seven distal) that were $1 \mathrm{~cm}$ in length and $1 \mathrm{~cm}$ apart. Electrode number three was positioned at the centre of the electrically active region of the diaphragm (EARdi) at functional residual capacity (FRC). The diaphragm CMAP elicited by bilateral magnetic stimulation of the phrenic nerves was simultaneously recorded from four electrode pairs. Pair one was created from electrodes one and three, pair two from electrodes two and four, pair three from electrodes three and five, and pair four from electrodes five and seven. Phrenic nerve stimulation was at residual volume $(\mathrm{RV}), \mathrm{FRC}, \mathrm{FRC}+1.0 \mathrm{~L}, \mathrm{FRC}+2.0 \mathrm{~L}$, and total lung capacity (TLC).

The CMAP recorded from pair one was least influenced by changes in lung volume and the amplitude was $2.41 \pm 0.39$ (mean $\pm \mathrm{sD}$ ), $2.60 \pm 0.27,2.64 \pm 0.29$, and $2.71 \pm 0.45 \mathrm{mV}$ at RV, FRC, FRC+1.0 L and FRC $+2.0 \mathrm{~L}$, respectively. At TLC the CMAP was more variable. The CMAP amplitude recorded from pair two increased with increasing lung volume and at FRC+2.0 $\mathrm{L}$ was 3.7 times larger than that at FRC. Pair four usually recorded substantially smaller CMAPs at all lung volumes.

This study shows that the diaphragm compound muscle action potential recorded from an oesophageal electrode just above the diaphragm is relatively stable over the lung volume range residual volume to functional residual capacity $+2.0 \mathrm{~L}$.
\end{abstract}

Eur Respir J 2000; 15: 1033-1038.

Dept of Respiratory Medicine and Allergy, Guy's, King's and St Thomas' School of Medicine, King's College Hospital, London, UK.

Correspondence: Y.M. Luo

Respiratory Muscle Laboratory

King's College Hospital

Bessemer Rd

London SE5 9PJ

UK

Fax: 442073463589

Keywords: Diaphragm compound muscle action potential

lung volume

magnetic stimulation

phrenic nerve

Received: August 31999

Accepted after revision December 151999
Measurement of the oesophageal diaphragm electromyography (EMG) could be an important technique to assess neural respiratory drive since it is less contaminated by other chest wall muscle activity $[1,2]$. Animal studies have shown a good correlation between the phrenic nerve neurogram and the diaphragm EMG [3]. The integrated EMG during voluntary breathing has been used to assess neural drive in humans [4]. However, accurate assessment of neural respiratory drive requires reliable recording of the diaphragm EMG that is not influenced by changes in lung volume. Many studies have addressed the effect of lung volume on the oesophageal diaphragm compound muscle action potential (CMAP). The studies performed in animals showed that change of lung volume had only a small effect on diaphragm CMAP amplitude $[5,6]$. In contrast, GANDEVIA and McKeNZIE [7] using a balloon stabilized oesophageal electrode to record the human diaphragm CMAP during supramaximal electrical stimulation (ES) at different lung volumes, reported that dia- phragm CMAP amplitude systematically increased with increasing lung volume. Consequently, the ability to quantify the oesophageal diaphragm EMG to assess neural respiratory drive was questioned.

Oesophageal electrode positioning is important for reliable recording of the diaphragm CMAP [5]. If a diaphragm CMAP can be recorded independent of change in lung volume, quantification of the oesophageal diaphragm CMAP [8] could be more useful in clinical practice, and the voluntary EMG may potentially become a useful clinical index of neuro-respiratory drive. In the present study a multipair oesophageal electrode was used, with careful control of positioning to ensure one recording electrode was at the centre of the electrically active region of the diaphragm (EARdi). The purpose of the study was to investigate the influence of lung volume on the amplitude of the diaphragm CMAP recorded from different electrode positions and to determine the optimal position for recording. 


\section{Methods}

\section{Subjects}

Six healthy volunteers (three males and three females) aged 26-36 yrs (mean age 31 yrs) participated in the study. All were free of neurological and respiratory disease. The study was approved by the ethics committee of King's College Hospital and all subjects gave their informed consent.

\section{Bilateral magnetic stimulation of the phrenic nerves at different lung volumes.}

Studies were performed with the subjects seated upright in a chair. Lung volume changes were measured with a spirometer (Ohio 840; AIRCO, Houston, TX, USA). Inspiratory capacity, expiratory reserve volume, and vital capacity were measured, with volumes referenced to functional residual capacity (FRC) which was taken as resting end-expiratory volume. Studies were performed at residual volume (RV), FRC, FRC+1.0 L, FRC+2.0 L, and total lung capacity (TLC). To do this the volume signal was displayed on a storage oscilloscope (Kikusui 5040 Kikusui Electronics Corporation, Yokohama, Japan) in front of the subject and operator. Subjects breathed to the appropriate lung volume and relaxed against a closed airway. Relaxation of the diaphragm was confirmed by the absence of diaphragm EMG recorded from oesophageal electrodes. Bilateral magnetic stimulation of the phrenic nerves was performed using two $43 \mathrm{~mm}$ figure of eight coils $(\mathrm{P} / \mathrm{N}$ 9784-00) powered by Magstim 200 stimulators (Magstim Co. Ltd., Whitland, Dyfed, UK). The coils were placed anterolaterally on each side of the neck over the phrenic nerves $[9,10]$. Five to seven supramaximal twitches were obtained at each lung volume. a)

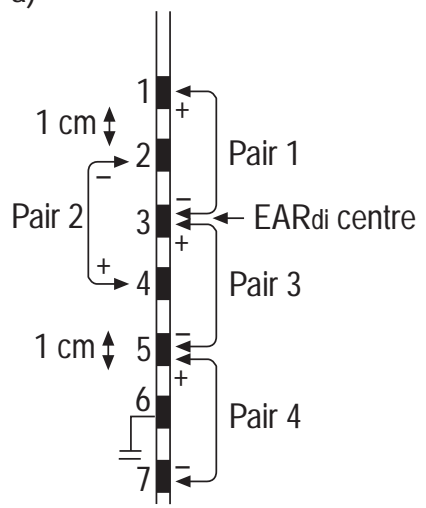

b)

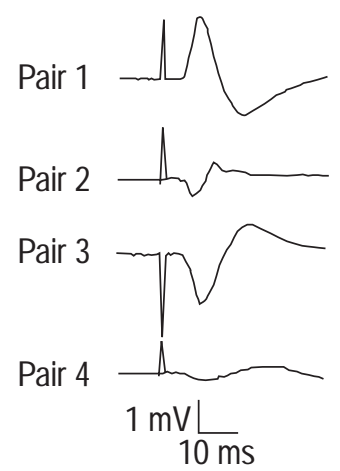

Fig. 1. - a) Oesophageal electrode configuration. The oesophageal electrode catheter consists of seven coils which form four recording electrode pairs. Each electrode is $1 \mathrm{~cm}$ in length and there is a $1 \mathrm{~cm}$ gap between electrodes. The interelectrode distance within an electrode pair is $3 \mathrm{~cm}$. Electrode three is placed at the centre of the electrically active region of the diaphragm (EARdi). b) compound muscle action potential (CMAP) recorded from the four electrode pairs at functional residual capacity. The signals from pairs one and three are opposite in polarity. Pair two only records a small CMAP since both electrodes are close to EARdi centre. Pair four records a small CMAP since the electrode is 3 $\mathrm{cm}$ away from the diaphragm. The signals shown are from one subject.
Recording of the compound muscle action potential

The oesophageal electrode catheter consisted of seven copper coils (number one being proximal and seven distal) $1 \mathrm{~cm}$ in length and $1 \mathrm{~cm}$ apart (fig. 1). The electrodes therefore created four electrode pairs, with electrode six connected to ground. Pair one consisted of electrodes one and three, pair two of electrodes two and four, pair three of electrodes three and five, and pair four of electrodes five and seven. Pairs one and three therefore shared a common electrode (number three). The recording pairs had an interelectrode distance of $3 \mathrm{~cm}$. The oesophageal electrode was passed through the nose and swallowed into the oesophagus. Electrode three was positioned at the centre of the EARdi at FRC based on the amplitude and opposite polarity of the diaphragm CMAP recorded from pairs one and three since CMAP shape is sensitive to electrode position. The position was further adjusted by observing the amplitude and polarity of the CMAP recorded from pair two, which was characterized by a small or absent CMAP since there is cancellation of the potential when both recording electrodes are equally close to the source of potential [7]. The catheter was moved slightly up (when the CMAP from pair two showed an initial positive waveform) or down (when the CMAP showed a negative waveform). The ideal position of the electrode was characterized by a large CMAP amplitude from pairs one and three, with opposite polarity, and only a small CMAP recorded from electrode pair two. After the electrode was optimally positioned, the catheter was taped at the nose. The diaphragm CMAP was recorded simultaneously from the multiple electrode pairs at different lung volumes. To study reproducibility four subjects were assessed on separate occasions.

EMG signals were amplified and band-pass filtered between $10 \mathrm{~Hz}$ and $10 \mathrm{kHz}$ (Magstim Co. Ltd.). The signals were then passed to a 12 bit analogue-to-digital converter (NB-MIO-16; National Instruments, Austin, TX, USA) and stored and displayed on a Macintosh Centris Computer running Labview 2.2 software (National Instruments). Each CMAP following stimulation could be viewed in real time.

\section{Additional studies}

To compare the $3 \mathrm{~cm}$ interelectrode distance with one of $5 \mathrm{~cm}$, as used by GANDEVIA and McKeNZIE [7], electrode number four was positioned at the centre of EARdi. The diaphragm CMAP was recorded from two pairs of electrodes, pair A (electrodes one and four) and pair B (electrodes two and four), simultaneously during bilateral phlenic nerve stimulation at different lung volumes in two subjects.

To compare the current results with those reported by GANDEVIA and McKenZIE [7] the current authors also studied two subjects with a balloon stabilized oesophageal electrode. The catheter consisted of one pair of electrodes, $1 \mathrm{~cm}$ in width and $5 \mathrm{~cm}$ apart. There was $1 \mathrm{~cm}$ between the lower electrode and the balloon $(2 \mathrm{~cm}$ in length and 4 $\mathrm{cm}$ in circumference). The balloon was positioned in the stomach, inflated with $15 \mathrm{~mL}$ air, and pulled back to wedge at the oesophagogastric junction. A $20 \mathrm{~g}$ weight was attached to the proximal end of the catheter to "stabilize" the electrode. 


\section{Data analysis}

The diaphragm CMAP was analysed off-line and the amplitude of the CMAP was measured from peak to peak. Only CMAPs with a constant shape and a stable baseline before and after stimulation were analysed to avoid the influence of artefacts resulting from electrocardiography (ECG) and oesophageal peristalsis. Results are expressed as the mean \pm SD. An analysis of variance (ANOVA) and paired t-test were used to assess the differences in diaphragm CMAP amplitude at different lung volumes. The variability of the CMAP amplitude at different lung volumes (from $\mathrm{RV}$ to $\mathrm{FRC}+2.0 \mathrm{~L}$ ) was evaluated by coefficient of variation $(\mathrm{CV})$. The relationship between pair $\mathrm{A}$ and pair B was assessed by regression. Significance was defined as $\mathrm{p}<0.05$.

\section{Results}

Good quality diaphragm CMAPs could always be recorded from pairs one and three during bilateral magnetic phrenic nerve stimulation and all CMAP signals obtained from pair one began with a predominantly positive wave, opposite to the signal from pair three, when stimulation was delivered at FRC. At FRC the maximal CMAP amplitude was always recorded from one of these two pairs. Pair two recorded a small CMAP at FRC, confirming that electrode three was at or close to the centre of the EARdi for all subjects (fig. 2). Pair four recorded a very small CMAP, as expected, since the electrodes were $3 \mathrm{~cm}$ away from the diaphragm. Similar results were obtained from four subjects on separate occasions.

\section{Electrode pair one}

The amplitude of the diaphragm CMAP recorded from pair one was relatively unaffected by lung volume changes, from $2.41 \pm 0.39 \mathrm{mV}$ at $\mathrm{RV}$ to $2.71 \pm 0.45 \mathrm{mV}$ at $\mathrm{FRC}+2.0 \mathrm{~L}$ ( $\mathrm{p}=\mathrm{NS}$; fig. 3). The $\mathrm{CV}$ was 10 (range $4-15$ ) from $\mathrm{RV}$ to $\mathrm{FRC}+2.0 \mathrm{~L}$. The polarity of the CMAP was always the same at different lung volumes (fig. 2). a)

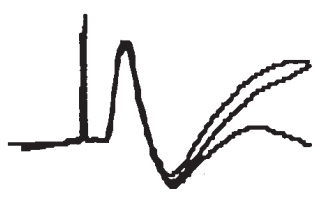

b)

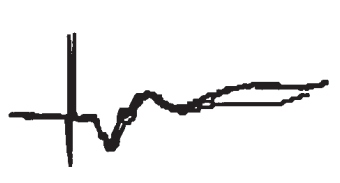

c)

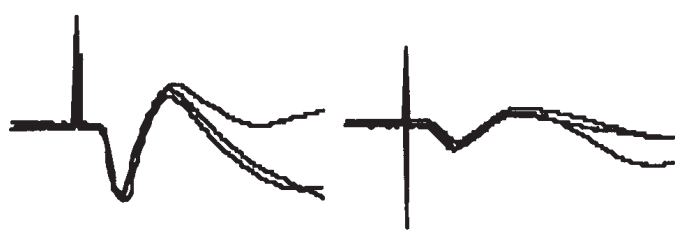

FRC
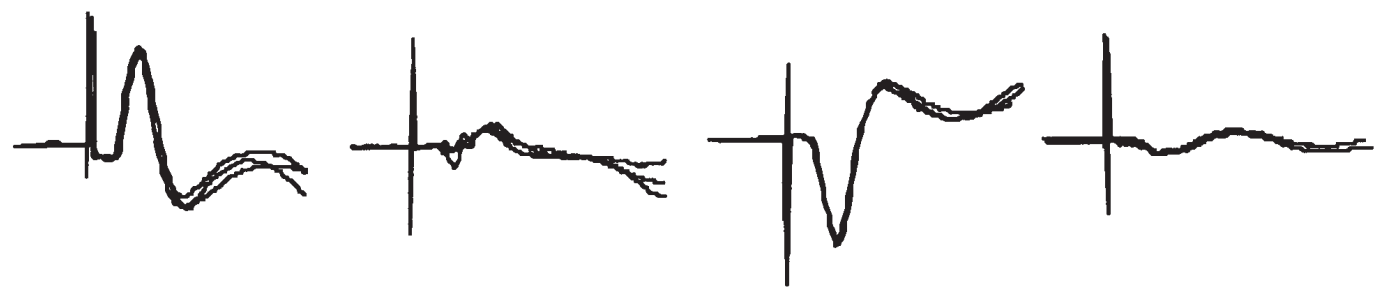

$\mathrm{FRC}+1.0 \mathrm{~L} \longrightarrow$
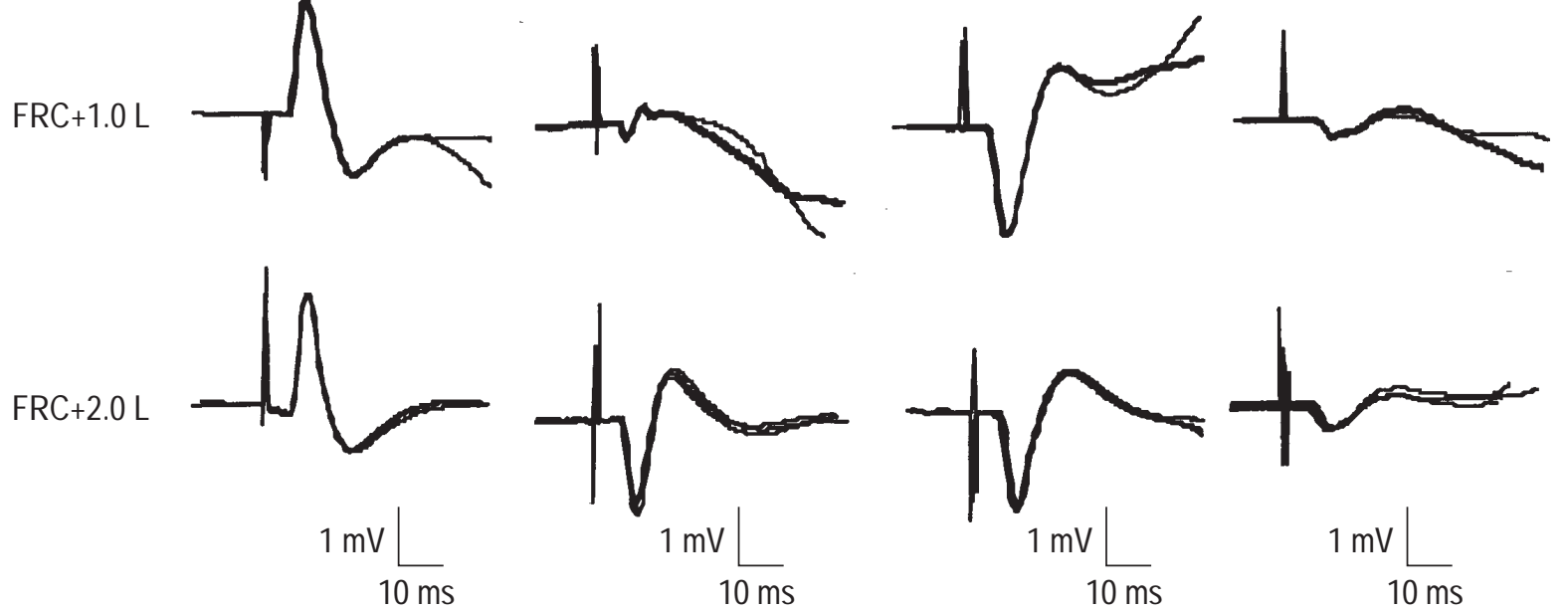

Fig. 2. - The diaphragm compound muscle action potential (CMAP) recorded from four electrode pairs at different lung volumes. The CMAP amplitude recorded from pair one (a) is relatively stable when lung volume changes from residual volume (RV) to functional residual capacity (FRC)+2.0 L. The CMAP amplitude recorded from pair two (b) increases when lung volume changes to FRC+2.0 L. The diaphragm CMAP recorded from pair four (d) is small even at high lung volumes. Data shown are from one subject. Three or four signals are superimposed over each over. c): the CMAP amplitude recorded from pair three. 


\section{Electrode pair two}

The CMAP amplitude systematically increased with increasing lung volume from FRC to FRC+2.0 L $(\mathrm{p}<0.01)$. The mean amplitude measured from FRC+1.0 L (2.03 $\mathrm{mV}$ ) was 2.8 times larger than that from FRC and the amplitude measured from FRC+2.0 L $(2.70 \mathrm{mV})$ was 3.7 times larger. The CMAP amplitude measured at FRC was usually smaller than at any other lung volume including RV (fig. 2).

\section{Electrode pair three}

The amplitude of the CMAP changed with lung volume. The polarity of the signal was the same at different lung volumes in all subjects, except one whose CMAP polarity altered when lung volume was at TLC. The variability of the diaphragm CMAP was greater than that from pair one with a CV of $25(9-47)$.

\section{Electrode pair four}

The CMAP amplitude significantly increased with increasing lung volume $(\mathrm{p}<0.05)$. The CMAP amplitude at $\mathrm{FRC}+2.0 \mathrm{~L}$ was up to eight times greater than that at RV (fig. 2).

The diaphragm CMAP amplitude summated from all recording pairs was more stable than that from individual pairs two, three and four when lung volume changed from $\mathrm{RV}$ to $\mathrm{FRC}+2.0 \mathrm{~L}$ but was not more stable than that from pair one. The CV (17 \pm 8$)$ for the summated CMAP, was larger than that $(10 \pm 4)$ from pair one.

In five subjects the CMAP polarity recorded from pairs one and three remained the same when lung volume changed from RV to TLC (fig. 2). This suggested that the EARdi centre at different lung volumes remained relatively close to the level of electrode three. For pair two,

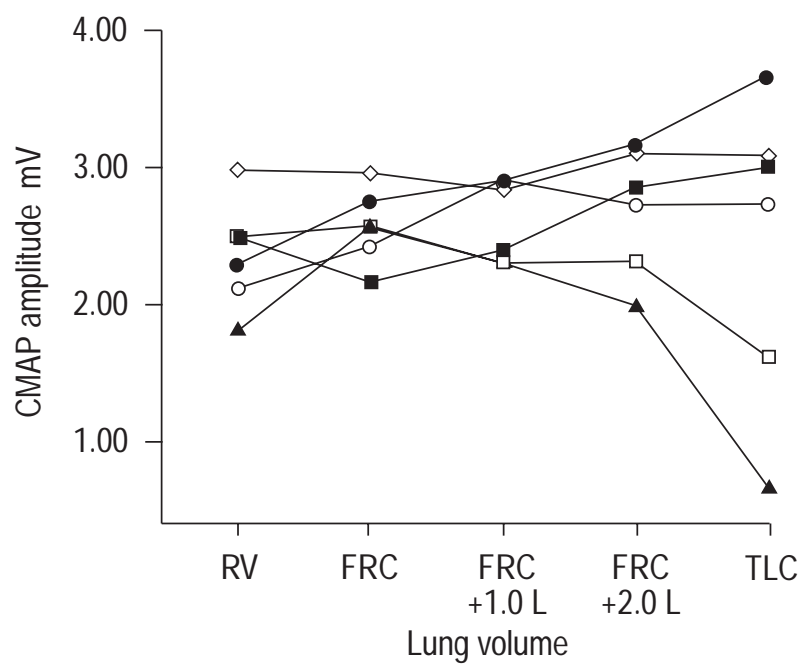

Fig. 3. - The diaphragm compound muscle action potential (CMAP) amplitude at different lung volumes recorded from pair one in each subject (each symbol represents one subject). The CMAP amplitude is relatively stable between residual volume (RV) and functional residual capacity $(\mathrm{FRC})+2.0 \mathrm{~L}$. The CMAP amplitude is considerably more variable at total lung capacity (TLC). in which the connection between the electrodes and the input terminals of the amplifier differed from the other pairs as shown in figure 1, a small CMAP amplitude was replaced with a large negative polarity CMAP when lung volume changed from low lung volumes to TLC. This suggested that the EARdi centre moved from the level of electrode three towards electrode four. In one subject the CMAP polarity recorded from pair three reversed at TLC. Therefore, in five of the six subjects the EARdi centre was unlikely to have moved $>1.5 \mathrm{~cm}$, assuming an unchanged perpendicular relationship between the oesophageal electrode and the crural diaphragm muscle fibres [11], and only in one subject could the movement have been greater.

\section{Additional studies}

There was a good correlation of CMAP amplitude between pair A and pair B at different lung volumes ( $\mathrm{r}=0.99$, $\mathrm{p}<0.001)$. The polarity of the CMAP recorded from both pairs was always the same at different lung volumes. The mean amplitude of the CMAP recorded from pair B was $90 \%$ of that from pair A.

The balloon stabilized electrode moved with respiration. The catheter moved up to $5 \mathrm{~cm}$ when lung volume changed from RV to TLC. The amplitude of the CMAP measured from the balloon stabilized electrode at FRC $(2.13 \mathrm{mV})$ was lower than that measured from an electrode $(2.80 \mathrm{mV})$ with the same configuration, but without a balloon, positioned at the EARdi centre. For the balloon stabilized electrode the amplitude of the CMAP increased with increasing lung volume. The amplitude of the CMAP increased $>2$ times from RV to TLC.

\section{Discussion}

The present study demonstrates that the influence of lung volume on the oesophageal diaphragm CMAP varies with the recording position. The diaphragm CMAP recorded from the pair whose lower electrode was at the centre of the EARdi was usually least sensitive to changes in lung volume. The results suggest that the diaphragm CMAP could be quantified even when there is a change in lung volume (e.g. patients with asthma) and that, potentially the voluntary EMG could also be reliably quantified.

To assess the effect of lung volume on the diaphragm CMAP, transcutaneous electrical phrenic nerve stimulation (ES) has previously been used. However, with ES it can be difficult to sustain supramaximal phrenic nerve stimulation $[10,12,13]$ especially when stimulation is performed bilaterally. Supramaximal stimulation is critical since the CMAP recorded from the oesophageal electrode is a summation from the left and right hemidiaphragms whose shape and polarity can be different. Therefore, slightly submaximal stimulation on one side may cause a significant change of the summated CMAP amplitude. Even with great care maximal stimulation may not be achieved constantly [13] since stimulating-electrode position is so critical. In contrast, with bilateral anterolateral magnetic stimulation the phrenic nerves can be reliably maximally stimulated $[9,10]$. The ability to easily achieve maximal phrenic nerve stimulation makes magnetic stimulation a useful investigative tool. 
To take account of diaphragm movement many investigators have used an oesophageal electrode catheter with a stabilizing balloon [7, 14]. However, ÖNAL et al. [15] found no difference between the voluntary EMG recorded by oesophageal electrodes with and without a stabilizing balloon. SCHWEITZER et al. [16] suggested that anchoring of oesophageal electrodes in man is unnecessary because high intraluminal pressure, generated by contraction of encircling fibres of the crural diaphragm and specialized smooth muscle of the wall of the lower oesophagus [17], should prevent significant displacement of a thin catheter. BECK et al. [11] speculated that balloon stabilization only serves to maintain the distance between the electrode and the oesophagus and questioned the usefulness of the balloon in controlling the distance between the electrode and the crus of the diaphragm. Gandevia and McKenzie [7], using fluoroscopy to observe electrode movement, found that the electrode moved $\sim 8 \mathrm{~cm}$ when lung volume changed from RV to TLC. This suggested that the electrode did not have a constant relationship to the oesophagus which is fixed in the thorax. Gandevia and McKenzie [7] also reported that with their balloon-stabilized electrode, with a $5 \mathrm{~cm}$ interelectrode distance, the signal polarity was not always the same. This could be because a balloon stabilized electrode does not always accurately track the source of the action potential. BECK et al. [11] observed that the EARdi centre moved only $1-4 \mathrm{~cm}$ based on the voluntary EMG signal polarity recorded from consecutive electrode pairs. In the present study it was found that in most subjects the EARdi centre moved $<1.5 \mathrm{~cm}$ from RV to TLC when the diaphragm was relaxed. The crus fibres encircle, and are approximately perpendicular to, the oesophagus $[11,18]$; and the contraction of the crus muscles grips the oesophagus and prevent gastro-oesophageal reflux rather than causing a descent of the crural diaphragm. In the current study it was observed that the catheters with stabilizing balloons moved up to $5 \mathrm{~cm}$, with deep inspiration, from RV, which was in agreement with the results reported by Gandevia and McKenzie [7]. However, the level of the EARdi centre did not change, based on the CMAP polarity from pairs one and three, at different lung volumes. The present authors speculate that the large movement of balloon stabilized catheters may be the result of diaphragm dome movement displacing the stomach and the balloon. The discrepancy between the catheter movement and the EARdi centre change suggests that balloon stabilized electrodes do not reliably record the CMAP from the crus part of the diaphragm.

One of the interesting findings of the present study was that the amplitude of the CMAP recorded from pair one did not significantly change with increasing lung volume up to FRC $+2.0 \mathrm{~L}$. This could be important when quantifying the diaphragm CMAP [8] in a clinical situation where performing twitches at the same lung volume may not be easy. The stability of the EMG, despite changes in lung volume, could also be physiologically and clinically important to assess respiratory neural output using oesophageal diaphragm EMG, which was frequently used in the 1970s [4, 15, 19] but less so after GANDEVIA and McKenZIE [7] suggested in 1986 that the oesophageal diaphragm EMG could not accurately reflect respiratory neural drive because of the considerable influence of lung volume on the diaphragm CMAP.
It is interesting to consider why the influence of lung volume on the diaphragm CMAP recorded from various positions is different. Changes in conductivity of the tissues surrounding the electrodes following change in lung volume has been suggested as an explanation of the changes of CMAP amplitude [5]. However, this mechanism could not be important in the present study since the electrode pairs were consecutive or overlapped each other and the influence of conductivity on each pair would be similar. Simple movement of the EARdi during changes in lung volume is not an adequate explanation because this would cause an increase in the CMAP measured from one electrode pair and a reduction in another, although it may partly explain the changes in CMAP amplitude recorded from pair two. It is possible that increasing lung volume changes the orientation of the diaphragm muscle fibres relative to the recording electrodes. When lung volume increases the muscle fibres that are approximately perpendicular to the electrode at low lung volume may become more parallel to the electrode and change CMAP amplitude. Since the diaphragm crus spans 2-3 cm [11] when relaxed, electrode three positioned at the EARdi can be maintained adjacent to the diaphragm even when the crus descends up to $1.5 \mathrm{~cm}$ at high lung volumes. This may partly explain why the CMAP amplitude from the upper electrode pair is not reduced whereas CMAP amplitude increases in the lower pairs.

DAUBENSPECK et al. [20] recorded the diaphragm EMG with a multipair electrode and summated the recordings from each channel. They found that the diaphragm EMG, after processing with their method, was more stable than when measured from a single channel. However, the oesophageal diaphragm CMAP amplitude depends not only on the radial distance but also on the fibre direction which may alter with changes in lung volume. Even when an electrode covers the whole oesophagogastric junction, the summated EMG amplitude may vary with changes in lung volume. Indeed, DAUBENSPECK et al. [20] found that the signal was relatively stable only for $\mathrm{FRC} \pm 1.0 \mathrm{~L}$. In the present study it was found that the mean amplitude summated from all recording pairs was more stable than that recorded separately from electrode pairs two, three and four. However, this summated CMAP amplitude was not more stable than that recorded from the upper pair (pair one).

In conclusion the influence of lung volume on the oesophageal diaphragm compound muscle action potential is affected by the recording electrode position. A bipolar electrode, one pole of which is positioned at the electrically active region of the diaphragm centre at functional residual capacity, is able to reliably record the diaphragm compound muscle action potential independent of lung volume over the range residual volume to functional residual capacity $+2.0 \mathrm{~L}$.

\section{References}

1. Luo YM, Polkey MI, Johnson LC, et al. Diaphragm EMG measured by cervical magnetic and electrical phrenic nerve stimulation. J Appl Physiol 1998; 85: 2089-2099.

2. Luo YM, Polkey MI, Lyall RA, Moxham J. Effect of brachial plexus coactivation on phrenic nerve conduction time. Thorax 1999; 54: 765-770.

3. Lourenco RV, Cherniack NS, Malm JR, Fishman AP. 
Nervous output from the respiratory center during obstructed breathing. J Appl Physiol 1966; 21: 527-533.

4. Lopata M, Evanich MJ, Lourenco RV. Quantification of diaphragmatic EMG response to $\mathrm{CO}_{2}$ rebreathing in humans. J Appl Physiol 1977; 43: 262-270.

5. Grassino AE, Whitelaw WA, Milic-Emili J. Influence of lung volume and electrode position on electromyography of the diaphragm. J Appl Physiol 1976; 40: 971-975.

6. Kim MJ, Druz WS, Danon J, Machnach W, Sharp JT. Effects of lung volume and electrode position on the oesophageal diaphragmatic EMG. J Appl Physiol 1978; 45: 392-398.

7. Gandevia SC, McKenzie DK. Human diaphragmatic EMG: changes with lung volume and posture during supramaximal phrenic stimulation. J Appl Physiol 1986; 60, $1420-1428$.

8. Luo YM, Lyall RA, Harris ML, Rafferty GF, Polkey MI, Moxham J. Quantification of the esophageal diaphragm EMG with magnetic phrenic nerve stimulation. Am J Respir Crit Care Med 1999; 160: 1629-1634.

9. Luo YM, Johnson LC, Polkey MI, et al. Diaphragm electromyogram with unilateral magnetic stimulation of the phrenic nerves. Eur Respir J 1999; 13: 385-390.

10. Mills GH, Kyroussis D, Hamnegard CH, Polkey MI, Green M, Moxham J. Bilateral magnetic stimulation of the phrenic nerves from an anterolateral approach. Am J Respir Crit Care Med 1996; 154: 1099-1105.

11. Beck J, Sinderby C, Lindstrom L, Grassino A. Influence of bipolar oesophageal electrode positioning on measurements of human crural diaphragm electromyogram. $J$ Appl Physiol 1996; 81: 1434-1449.
12. Bellemare F, Bigland-Ritchie B. Assessment of human diaphragm strength and activation using phrenic nerve stimulation. Respir Physiol 1984; 58: 263-277.

13. Bellemare F, Bigland-Ritchie B. Central components of diaphragmatic fatigue assessed by phrenic nerve stimulation. J Appl Physiol 1987; 62: 1307-1316.

14. Smith J, Bellemare F. Effect of lung volume on in vivo contraction characteristics of human diaphragm. $J$ Appl Physiol 1987; 62: 1893-1900.

15. Önal E, Lopata M, Evanich MJ. Effects of electrode position on oesophageal diaphragmatic EMG in humans. $J$ Appl Physiol 1979; 47: 1234-1238.

16. Schweitzer TW, Fitzegerald JW, Bowden JA, LynnDavies P. Spectral analysis of human diaphragm electromyogram. J Appl Physiol 1979; 46: 152-165.

17. Salmons S. Muscle. In: Bannister LH, Berry MM, Collins P, Dyson M, Dussek JE, Ferguson MWJ, eds. Gray's anatomy. 38 Edn. New York, NY, USA, Churchill Livingstone, 1995; pp. 737-900.

18. Beck J, Sinderby C, Lindstrom L, Grassino A. Diaphragm interference EMG and compound muscle action potentials: effects of chest wall configuration. J Appl Physiol 1997; 82: 520-530.

19. Lopata M, Evanich MJ, Lourenco RV. Relationship between mouth occlusion pressure and electrical activity of the diaphragm: effect of flow-resistive loading. Am $J$ Respir Crit Care Med 1977; 116: 449-455.

20. Daubenspeck JA, Leiter JC, McGovern JF, Knuth SL, Kobylarz EJ. Diaphragmatic electromyography using a multiple electrode array. J Appl Physiol 1989; 67: 15251534. 